\title{
Standortbestimmung bei «emerging» kardiovaskulären Risikofaktoren
}

\author{
Arbeitsgruppe Lipide und Atherosklerose (AGLA) der Schweizerischen Gesellschaft für Kardiologie*
}

\section{Einleitung}

In den letzten Jahren sind zahlreiche Daten zu möglichen neuen Risikofaktoren gewonnen worden. Diese werden in Abgrenzung zu den etablierten klassischen Risikofaktoren oft als «emerging» bezeichnet. Den aktuellen praxisrelevanten Erkenntnisstand dazu hat die Arbeitsgruppe Lipide und Atherosklerose der Schweizerischen Gesellschaft für Kardiologie für die Gebiete Genetik, Ernährung, Entzündung und biochemische Parameter (Homocystein, C-reaktives Protein, D-Dimere) für die Ärzteschaft in Empfehlungen aufgearbeitet. Damit wird bezweckt, den praktizierenden Kollegen in einem teilweise verwirrenden Dickicht von auch kommerziellen Angeboten ein sinnvolles Vorgehen zu empfehlen. Die Empfehlungen sind in Tabelle 1 und den folgenden fünf Zusammenfassungen wiedergegeben, die sich jede mit einem Risikofaktor befassen. Die vollständigen Artikel mit ausführlicher Beschreibung des wissenschaftlichen Hintergrunds und den zu den Empfehlungen führenden Überlegungen sind in der Zeitschrift Kardiovaskuläre Medizin 2004, Jahrgang 7, Nummern 1 und 2, erschienen. Sie können auch auf der Homepage www.agla.ch vollständig abgerufen werden.

\section{Die Erarbeitung der Empfehlungen}

Die Empfehlungen der Arbeitsgruppe Lipide und Atherosklerose (AGLA) richten sich an Grundversorger und Spezialisten, die Patienten mit potentiell erhöhtem kardiovaskulärem Risiko betreuen. Bereits 1995 wurden seitens der AGLA Empfehlungen zur Rolle verschiedener kardiovaskulärer Risikofaktoren publiziert [1]. Die Empfehlungen zum Risikofaktor Cholesterin wurden im Jahre 2000 als von der FMH anerkannte Empfehlungen publiziert [2] und im Jahre 2003 teilweise revidiert. Empfehlungen der AGLA zur Prävention der Atherosklerose werden zurzeit erarbeitet und in den nächsten Monaten veröffentlicht werden.

Das Ziel der gegenwärtigen Empfehlungen ist nun, über aktuelle Entwicklungen bei einigen «emerging» kardiovaskulären Risikofaktoren zu informieren. Bei diesen werden gegenwärtig Evidenz, Relevanz sowie Interventionen in der Literatur breit und kritisch diskutiert. Zudem werden von verschiedenen Anbietern diagnostische und therapeutische Massnahmen angepriesen.

Bei der Ausarbeitung der Empfehlungen bemühte sich die AGLA, ein möglichst breites Spektrum von Fachwissen und praktischer Erfahrung zu berücksichtigen. Die Ausarbeitung der Empfehlungen erfolgte unter der Leitung von Prof. Edouard Battegay, Basel, und wurde vom Vorstand der AGLA (Prof. Roger Darioli, Lausanne; PD Dr. Richard James, Genf; Prof. Ulrich Keller, Basel; Prof. Georg Noll, Zürich; Prof. Giorgio Noseda, Mendrisio [Präsident der AGLA]; Prof. Walter F. Riesen, St. Gallen), einem Vertreter der Schweizerischen Herzstiftung (PD Dr. med. Rubino Mordasini, Bern) sowie Vertretern der Schweizerischen Gesellschaft für Allgemeinmedizin (Dr. med. Brigitte Saner, Olten) und der Schweizerischen Gesellschaft für Innere Medizin (Dr. med. Christian Graf, Zürich) von Beginn weg begleitet. Für die Ausarbeitung der einzelnen Kapitel zu den Risikofaktoren wurden ausgewiesene Fachexperten zugezogen (Genetik: PD Dr. André R. Miserez, Bruderholz. Ernährung: PD Dr. Paolo Suter, Zürich; Prof. Roger Darioli, Lausanne; Prof. Giorgio Noseda, Mendrisio; PD Dr. Georg Schulthess, Zürich. Homocystein: Prof. Bryan Fowler, Basel. D-Dimer: Dr. Wolfgang Korte, St. Gallen; Prof. Walter F. Riesen, St. Gallen. CRP und Infektion: Prof. François Mach, Genf). Die Kapitel zu den jeweiligen Risikofaktoren wurden sowohl vom Vorstand der AGLA (siehe oben) als auch vom Redaktionsausschuss der Empfehlungen (Prof. Edouard Battegay, Basel [Leitung]; Prof. Roger Darioli, Lausanne; Dr. Christian Graf, Zürich; PD Dr. André R. Miserez, Bruderholz; Prof. Walter Riesen, St. Gallen; Dr. Brigitte Saner, Olten; PD Dr. Georg Schulthess, Zürich) gegengelesen und kritisch evaluiert. Schliesslich wurden die Dokumente vom Redaktionsausschuss zur Publikation finalisiert, durch den Vorstand der AGLA genehmigt und durch die Schweizerische Gesellschaft für Kardiologie anerkannt. 
Der Vorstand der AGLA wird durch die Mitgliederversammlung der AGLA gewählt, und die Leitung der Ausarbeitung der Empfehlungen und der Redaktionsausschuss wurden durch den Vorstand der AGLA bestimmt. Die AGLA steht allen offen, die sich für Risikofaktoren der Atherosklerose interessieren, insbesondere Grundversorgern, Spezialisten und Wissen- schaftlern. Industrievertreter mit potentiellen Interessenkonflikten können nicht Einzelmitglieder der AGLA sein. Die Arbeit in sämtlichen Gremien (AGLA-Vorstand, Leitung Ausarbeitung der Empfehlungen, Redaktionsausschuss, Schreiben der Unterkapitel) war und ist freiwillig und unbezahlt (keine Sitzungsspesen, keine Honorare). Reisespesen zu den Sitzungen

Tabelle 1

Standortbestimmung bei «emerging» kardiovaskulären Risikofaktoren: Empfehlungen für die Praxis

\begin{tabular}{|c|c|c|c|c|}
\hline Risikofaktor & $\begin{array}{l}\text { Evidenz } \\
\text { als Risikofaktor }\end{array}$ & $\begin{array}{l}\text { Screening } \\
\text { aller Personen }\end{array}$ & $\begin{array}{l}\text { Abklärungen } \\
\text { bei Risikogruppen }\end{array}$ & Behandlungsempfehlungen \\
\hline \multirow[t]{5}{*}{ Genetik } & \multirow[t]{5}{*}{ ja } & \multirow[t]{5}{*}{ ja (Familienanamnese) } & $\begin{array}{l}\text { ja: genetische Tests bei: } \\
\text { manifester Atherosklerose bei } \\
\text { erstgradig verwandten Männern }<55 \text {, } \\
\text { Frauen }<65 \text { Jahren }\end{array}$ & $\begin{array}{l}\text { ja: aggressive Cholesterinsenkung } \\
\text { (Familienabklärung) }\end{array}$ \\
\hline & & & $\begin{array}{l}\text { Cholesterin }>8 \mathrm{mmol} / \mathrm{l} \\
\text { oder Triglyzeride }>5 \mathrm{mmol} / \mathrm{l}\end{array}$ & \\
\hline & & & $\begin{array}{l}\text { erstgradig Verwandten von Patienten } \\
\text { mit familiären Formen von Hyper- } \\
\text { lipoproteinämien ( } 50 \% \text { Risiko bei } \\
\text { autosomal-dominantem Erbgang) }\end{array}$ & \\
\hline & & & tendinösen und palmaren Xanthomen & \\
\hline & & & $\begin{array}{l}\text { Hypercholesterinämie } \\
\text { (Cholesterin }>6,5 \mathrm{mmol} / \mathrm{l}) \text { bereits } \\
\text { im Jugend- oder Kindesalter }\end{array}$ & \\
\hline \multicolumn{5}{|l|}{ Ernährung } \\
\hline Übergewicht/Adipositas & ja & $\begin{array}{l}\text { ja (Ernährungsanamnese, } \\
\text { Gewichtskontrolle) }\end{array}$ & $\begin{array}{l}\text { ja (Ernährungsanamnese, } \\
\text { Gewichtskontrolle) }\end{array}$ & $\begin{array}{l}\text { ja: Gewichtsreduktion oder } \\
\text { zumindest Stabilisierung }\end{array}$ \\
\hline Ernährungsmuster & ja & ja (Ernährungsanamnese) & ja (Ernährungsanamnese) & ja, siehe Tabelle 2 \\
\hline Alkohol & (ja) & $\begin{array}{l}\text { nein (ausser übermässiger } \\
\text { Konsum) }\end{array}$ & nein (ausser übermässiger Konsum) & $\begin{array}{l}\text { Alkoholkonsum nicht empfehlen, } \\
\text { aber moderate Einnahme tolerieren }\end{array}$ \\
\hline \multicolumn{5}{|l|}{ Nährstoffsupplemente } \\
\hline $\begin{array}{l}\text { Vitamine und } \\
\text { Spurenelemente }\end{array}$ & nein & nein & nein & $\begin{array}{l}\text { nein, bei Hyperhomocysteinämie: } \\
\text { siehe dort }\end{array}$ \\
\hline Fischölkapseln & ja & nein & ja (Ernährungsanamnese) & $\begin{array}{l}\text { ja: in der Sekundarprävention bei } \\
\text { fehlender Fischzufuhr oder anderweitig } \\
\text { nicht kontrollierbarer Hypertriglyzeridämie }\end{array}$ \\
\hline Functional Food & nein & nein & nein & nur in ausgewählten Fällen \\
\hline \multirow[t]{4}{*}{$\begin{array}{l}\text { Homocystein } \\
\text { (Total-Homocystein; } \\
\text { tHcy) }\end{array}$} & \multirow[t]{4}{*}{ ja } & \multirow[t]{4}{*}{ nein } & $\begin{array}{l}\text { ja: Bestimmung tHcy bei: } \\
\text { persönlicher oder familiärer } \\
\text { Anamnese von frühzeitiger Athero- } \\
\text { sklerose }\end{array}$ & $\begin{array}{l}\text { ja: für Bevölkerung allgemein: } \\
\text { tägliche Einnahme von frischen Früchten } \\
\text { und Gemüsen; Sistieren des Rauchens }\end{array}$ \\
\hline & & & $\begin{array}{l}\text { Atherosklerose ohne genügend } \\
\text { erklärende Ursache }\end{array}$ & $\begin{array}{l}\text { Sekundärprävention: bei tHcy }>15 \mu \mathrm{mol} / \mathrm{l} \text { : } \\
\text { Zielwert }<10 \mu \mathrm{mol} / \mathrm{l}\end{array}$ \\
\hline & & & \multirow[t]{2}{*}{$\begin{array}{l}\text { positiver Familienanamnese } \\
\text { für erhöhte tHcy; Messung } \\
\text { bei Kindern }>10 \text { Jahre }\end{array}$} & $\begin{array}{l}\text { Folsäure p. o. } 1 \mathrm{mg} / \mathrm{d}+ \\
\text { Vitamin } \mathrm{B}_{6} \text { p. o. } 40 \mathrm{mg} / \mathrm{d}+ \\
\text { Cyanocobalamin p. } 0.30 \mu \mathrm{g} / \mathrm{d} \text {. } \\
\text { Kontrolle nach } 1 \text { Monat: tHcy-Spiegel }\end{array}$ \\
\hline & & & & $\begin{array}{l}\text { bei Nichtansprechen auf Therapie } \\
\text { sowie bei mässiger }(30-100 \mu \mathrm{mol} / \mathrm{I}) \\
\text { und schwerer }(>100 \mu \mathrm{mol} / \mathrm{I}) \\
\text { Hyperhomocysteinämie: } \\
\text { Überweisung an den Spezialisten }\end{array}$ \\
\hline D-Dimer & ja & nein & nein & nein \\
\hline $\begin{array}{l}\text { CRP (high sensitivity } \\
\text { CRP; hs-CRP) }\end{array}$ & ja & nein & nein & nein \\
\hline Infektion & ja & nein & nein & nein \\
\hline
\end{tabular}


wurden von der AGLA beglichen. Sponsoren waren während keiner Phase an der Ausarbeitung des Inhaltes der Empfehlungen beteiligt. Die Kosten der Erarbeitung der Empfehlung (Reisekosten, Miete Sitzungszimmer, Druckkosten, Korrespondenz usw.) wurden durch keine Sponsoren direkt gestützt. Allerdings benützte die AGLA Gewinne, die durch Kongressveranstaltungen erzielt worden waren, um die Kosten zu decken. Kongresse der AGLA (das «Atherosklerose Update Meeting» und das «Lipidologen-Treffen») sind durch Beiträge von Sponsoren sowie zu einem kleineren Teil durch Beiträge von Kongressteilnehmern gedeckt und haben in den letzten Jahren zu kleinen Gewinnen geführt, die für dieses und andere Projekte genützt werden.

\section{Literatur}

1 Rolle von Risikofaktoren für die koronare Herzkrankheit. Empfehlungen der Arbeitsgruppe Lipide und Atherosklerose der Schweizerischen Gesellschaft für Kardiologie. Schweiz Ärztezeitung 1995;76:497-507.

2 Battegay E, Bertel O, Darioli R, Gutzwiller F, Keller U, Nigg C, et al. Empfehlungen 1999 zur Behandlungsindikation des Risikofaktors Cholesterin. Schweiz Ärztezeitung 2000; 81:2139-43.

\section{Genetische Risikofaktoren für kardiovaskuläre Krankheiten}

\section{A. R. Miserez}

Korrespondenz:

PD Dr. André R. Miserez

Kardiovaskuläre Risikosprechstunde Medizinische Universitätsklinik Kantonsspital Bruderholz

CH-4101 Bruderholz

E-Mail: andre-r.miserez@unibas.ch

\section{Wissenschaftliche Grundlagen}

Spezifische Genvarianten oder Gendefekte können das Risiko für die Entstehung einer Atherosklerose massiv fördern und damit auch die Inzidenz kardiovaskulärer Komplikationen deutlich erhöhen. Zahlreiche Defekte wurden in Genen entdeckt, die beispielsweise am Cholesterinstoffwechsel beteiligt sind. Genetische Lipidstoffwechselstörungen führen typischerweise bei Männern bereits vor dem 55. bzw. bei Frauen vor dem 65. Altersjahr zu atherosklerotischen Ereignissen. $\mathrm{Zu}$ diesen Störungen zählen das familiär-defektive Apo B-100 (FDB), die familiäre Dysbetalipoproteinämie (FDL), die familiäre Hypercholesterinämie (FHC), die familiärkombinierte Hyperlipidämie $(\mathrm{FCH})$, die schweren polygenen Hypercholesterinämien sowie weitere seltene Erkrankungen.

Eine frühzeitige Entdeckung und Behandlung dieser Hyperlipoproteinämien kann Myokardinfarkte verhindern. In Frühstadien können klinische Zeichen und Symptome fehlen. Wichtig ist deshalb die Familienanamnese bezüglich kardiovaskulärer Erkrankungen. Eine molekulargenetische Analyse kann gewisse Störungen bestätigen oder ausschliessen und ist bei der Abklärung weiterer Familienmitglieder sehr hilfreich. Heutzutage können mehrere dieser Defekte (z. B. FDB, FDL und FHC) routinemässig auf molekularer Basis diagnostiziert werden, andere häufige Störungen (z.B. FCH) hingegen nicht.

\section{Abklärung von Risikopatienten}

Obwohl FDB, FDL und FHC relativ häufig vorkommen (bei ungefähr 0,7\% der Allgemeinbevölkerung), ist die Wahrscheinlichkeit, bei einem Patienten ohne entsprechende Familienanamnese eine dieser Mutationen zu entdecken, doch relativ gering. Genetische Routinetests zur Erkennung dieser Lipidstoffwechselstörungen sind daher in einer unselektionierten Bevölkerungsgruppe nicht zu empfehlen. Bestehen dagegen eines oder mehrere der folgenden Zeichen, ist die Wahrscheinlichkeit, die Diagnose FDB, FDL oder FHC stellen zu können, sehr stark erhöht:

- manifeste Atherosklerose bei Patient/Patientin oder bei erstgradigen Verwandten (Männer $<55$ Jahre, Frauen $<65$ Jahre);

- erstgradig Verwandte von Patienten mit familiären Formen von Hyperlipoproteinämien;

- tendinöse oder palmare Xanthome;

- massive Hypercholesterinämie oder Hypertriglyzeridämie im Erwachsenenalter (Cholesterin $>8 \mathrm{mmol} / \mathrm{l}$ oder Triglyzeride $>5$ $\mathrm{mmol} / \mathrm{l})$.

\section{Molekulargenetische Tests}

Ein Screening soll anhand der obengenannten familienanamnestischen und phänotypischen Untersuchungen erfolgen. Die Identifikation 
eines betroffenen Patienten ermöglicht dann bei weiteren betroffenen Familienmitgliedern eine Diagnose, bevor sich der Phänotyp manifestiert (Frühdiagnose). Die weitere Abklärung der Familie und die Frühdiagnose mit Hilfe genetischer Untersuchungen ist deshalb wichtig, weil bei mindestens der Hälfte der betroffenen Familienmitglieder schon vor dem 50. Lebensjahr atherosklerosebedingte fatale Komplika- tionen zu erwarten sind. Der molekulargenetische Nachweis erlaubt diese Frühdiagnose; die Durchführung ist nur einmal im Leben notwendig. Beim genetischen Nachweis der Störung muss allerdings durch den Spezialisten abgewogen werden, ob die individuelle Risikosituation des Patienten nur zu intensivierten phänotypischen Untersuchungen oder zur Behandlung führen soll.

\title{
Ernährung und kardiovaskuläres Risiko
}

\author{
P. M. Suter, R. Darioli, G. Noseda, G. Schulthess
}

\section{Kardioprotektives Ernährungsmuster}

In den letzten Jahren hat das Interesse an der Ernährung als Ganzem sowie an ihren einzelnen nutritiven und nicht-nutritiven Komponenten als Faktoren der Herz-Kreislauf-Prävention deutlich zugenommen. Epidemiologische Studien haben gezeigt, dass weniger einzelne Komponenten der Ernährung als vielmehr Ernährungsmuster für die kardioprotektiven Effekte verantwortlich sind. Aufgrund verschiedenster Studien ist ein kardioprotektives Ernährungsmuster charakterisiert durch eine bedarfsgerechte Energiezufuhr (d.h. Beibehaltung eines normalen Körpergewichts), einen hohen Konsum an Früchten und Gemüse, eine Modifikation der Fettzufuhr (Reduktion der Zufuhr an gesättigten Fetten, Ersetzen der gesättigten Fette durch einfach und mehrfach ungesättigte Fette), einen vermehrten Fischkonsum sowie eine höhere Zufuhr an «komplexen», nahrungsfaserreichen Kohlenhydratprodukten und eine verminderte Zufuhr an «einfachen», raffinierten Kohlenhydraten. Die ideale Zusammensetzung der Ernährung zur Prävention chronischer Erkrankungen ist in Tabelle 2 zusammengefasst.

\section{Ernährungsempfehlungen}

Eine Ernährungsempfehlung ist stets individuell $\mathrm{zu}$ modifizieren. Parameter wie Alter, Gewicht, körperliche Aktivität, Serum-Lipide, kardiovaskuläres Risiko oder genetisch determinierte Stoffwechselstörungen sollen berücksichtigt werden. Die wichtigsten Grundsätze ernährungsmedizinischer Empfehlungen sind als tabellarische Übersicht zusammengefasst.
Tabelle 2

WHO-Empfehlungen der Energie- und Substratzufuhr.

\begin{tabular}{|c|c|}
\hline Nährstoff & Zufuhr-Empfehlung \\
\hline Gesamtfett & $\begin{array}{l}15-30 \% \\
\text { der Energiezufuhr }\end{array}$ \\
\hline gesättigte Fette & $\begin{array}{l}<10 \% \\
\text { der Energiezufuhr }\end{array}$ \\
\hline mehrfach ungesättigte Fette & $\begin{array}{l}3-7 \% \\
\text { der Energiezufuhr }\end{array}$ \\
\hline Eiweiss & $\begin{array}{l}\text { 10-15\% } \\
\text { der Energiezufuhr }\end{array}$ \\
\hline \multicolumn{2}{|l|}{ Kohlenhydrate } \\
\hline komplexe Kohlenhydrate* & $\begin{array}{l}55-75 \% \\
\text { der Energiezufuhr }\end{array}$ \\
\hline einfache Kohlenhydrate** & $\begin{array}{l}<10 \% \\
\text { der Energiezufuhr }\end{array}$ \\
\hline \multicolumn{2}{|c|}{$\begin{array}{l}\text { * d.h. nahrungsfaserreiche Kohlenhydratprodukte } \\
\text { ** d.h. raffinierte Kohlenhydrate }\end{array}$} \\
\hline
\end{tabular}

Die Wahl der idealen ernährungsmedizinischen Strategie ist nicht immer einfach, zumal das Antwortmuster in Abhängigkeit von den obenerwähnten Faktoren sehr unterschiedlich sein kann. Im Falle einer familiären kombinierten Hyperlipidämie muss beispielsweise vollständig auf Alkohol verzichtet werden, da es sonst zu einem beträchtlichen Anstieg der Triglyzeride kommen kann.

Das Thema Ernährung ist heute ein grosser Renner und entsprechend oft sind viele Empfehlungen medizinisch nicht vertretbar. Vor unkontrollierten Diäten mit beispielsweise drastischen Gewichtsveränderungen muss konsequent gewarnt werden, da diese gesundheitsschädigend sein können. 
Eine kardioprotektive Ernährung, wie in Tabelle 3 zusammengefasst, sollte während einer möglichst langen Lebensperiode gewährleistet sein, am besten bereits ab der Kindheit. Die
Massnahmen haben Gültigkeit für die Primärund Sekundärprävention von Herz-KreislaufErkrankungen.

Tabelle 3

Eine Ernährungsempfehlung ist stets individuell zu modifizieren. Parameter wie Alter, Gewicht, körperliche Aktivität, Serum-Lipide, kardiovaskuläres Risiko oder genetisch determinierte Stoffwechselstörungen sollen berücksichtigt werden. Das aufgeführte Schema ermittelt eine Basis hierzu. Warnung: Unkontrollierte Diäten mit z.B. drastischen Gewichtsveränderungen können gesundheitsschädigend sein.

generelle Empfehlung

Halten eines normalen Körpergewichts

oder im Falle von Übergewicht / Fettleibigkeit:

Stabilisation, wann immer möglich, Gewichtsreduktion

Durch eine Ernährung mit hoher Variabilität, entsprechend dem individuellen

Energiebedarf, wobei eine hohe körperliche Aktivität empfehlenswert ist

\section{Konsum fördern}

Täglich frische Früchte und Gemüse

Komplexe Kohlenhydrate (Vollkornprodukte, Vollreis usw.)

Fisch

Magermilchprodukte

Soja, Nüsse, Tee

Moderater Konsum

Einfache Kohlenhydrate (Weissbrot, Frühstückszerealen usw.)

Vollmilchprodukte

Pflanzliche Öle (nicht erhitzen, erst nach dem Kochen beifügen)

Fleisch, Salz

Moderater Alkoholkonsum ist zu tolerieren (cave Suchtpotential)

Konsum reduzieren

Fettreiche Speisen (Würste, Pommes frites, Saucen, Patisserie, Torten usw.)

Supplemente: antioxidative Vitamine, Spurenelemente

nein

Folsäure bei therapiebedürftiger Hyperhomocysteinämie

Fischölkapseln

nein

Fischölkapseln in der Sekundärprävention bei fehlender Fischzufuhr oder anderweitig

nicht kontrollierbarer Hypertriglyzeridämie

Functional Food, z. B. mit pflanzlichen Sterolen angereicherte Margarine

nein 


\section{Homocystein als kardiovaskulärer Risikofaktor}

B. Fowler

\author{
Epidemiologie und prognostische \\ Bedeutung
}

Ein leichter Anstieg des Totalhomocysteins (tHcy) im Nüchternzustand (15-30 $\mu \mathrm{mol} / \mathrm{l})$ oder nach Methionin-Belastung gilt als unabhängiger Risikofaktor für das Auftreten einer Atherosklerose. In Kombination mit anderen Risikofaktoren führen erhöhte Homocysteinwerte synergistisch (z.B. bei gleichzeitiger arterieller Hypertonie) oder additiv (z.B. bei gleichzeitig erhöhtem Cholesterinspiegel) zu einem Anstieg des Atheroskleroserisikos. Bei ungefähr 10\% aller Personen der Allgemeinbevölkerung und bei etwa 30\% aller Patienten mit Gefässkrankheiten werden erhöhte tHcy-Werte gemessen. Die Risikozunahme für die Entstehung einer koronaren Herzkrankheit bei einem tHcyAnstieg um $5 \mu \mathrm{mol} / \mathrm{l}$ ist vergleichbar mit derjenigen einer Cholesterinkonzentrationserhöhung um $0,5 \mathrm{mmol} / \mathrm{l}$.

\section{Ätiologie und Pathophysiologie}

Homocystein entsteht aus der Aminosäure Methionin. Die weitere Metabolisierung er folgt unter Beteiligung dreier Schlüsselenzyme: Methioninsynthase (MS), 5,10-Methylentetrahydrofolatreduktase (MTHFR) und Cystathionin-, -Synthase (CBS). Zu den Ursachen für leicht erhöhte Homocysteinspiegel zählen genetische und nutritive Faktoren, die zueinander in Wechselwirkung treten. So kann die Hyperhomocysteinämie familiär bedingt sein und zusätzlich durch Folsäure, Vitamin B12 und B6 beeinflusst werden. Der 677C/T-Polymorphismus des MTHFR-Gens (Homozygotie in etwa $10 \%$ der Bevölkerung in Europa) ist bei folatarmer Nahrung mit geringfügigen Zunahmen der Homocysteinkonzentrationen assoziiert.

\section{Primärprävention}

Durch Verabreichung von Folsäure als Monotherapie oder in Kombination mit Vitamin B6 und/oder B12 kann ein erhöhter Homocysteinspiegel gesenkt werden. Einige Autoren empfehlen, Hyperhomocysteinämien zu therapieren, wenn die tHcy-Spiegel oberhalb der oberen Normgrenze ( $15 \mu \mathrm{mol} / \mathrm{l})$ liegen, andere bei tHcyWerten über $10 \mu \mathrm{mol} / \mathrm{l}$. Allerdings existieren keine zuverlässigen Daten, die den Vorteil eines Screenings in der Allgemeinbevölkerung bzw. einer Behandlung leicht erhöhter tHcy-Werte in der Primärprävention belegen. In den USA wird zwar seit kurzem das Mehl mit Folsäure angereichert und allgemeine homocysteinsenkende Massnahmen sind generell zu empfehlen (tägliche Einnahme von frischen Früchten und Gemüse sowie das Sistieren des Rauchens).

\section{Empfehlungen in der kardiovaskulären Sekundärprävention und bei Hyper- homocysteinämien}

Eine Bestimmung des Homocysteins im Plasma empfiehlt sich in der Sekundärprävention bei frühzeitig auftretender Atherosklerose ohne genügend erklärende Ursache sowie bei Personen, in deren Familie erhöhte tHcy-Werte bekannt sind (Messung bei Kindern ab 10 Jahren). Die Bestimmung des tHcy-Spiegels sollte nach nächtlichem Fasten erfolgen. Die korrekte präanalytische und analytische Handhabung der Blutproben ist von kritischer Bedeutung. Bei Personen mit erhöhten tHcy-Werten (oberhalb der für die jeweilige Altersgruppe geltenden Normgrenze bzw. > 15 umol/l) soll in der Sekundärprävention eine Therapie durchgeführt werden. Ein Vitamin-B12-Mangel sowie andere Grundleiden, wie beispielsweise Nierenerkrankungen, müssen ausgeschlossen werden. Analysen von Polymorphismen, die mit dem Homocysteinmetabolismus assoziiert sind, werden zurzeit nur zu Forschungszwecken durchgeführt. Bei Patienten mit Verdacht auf familiäre Hyperhomocysteinämie sind Familienuntersuchungen einschliesslich der Testung von Kindern angezeigt.

\section{Praktische Durchführung der Therapie}

Bei nachgewiesener therapeutischer Indikation erhält der Patient täglich per os $1 \mathrm{mg}$ Folsäure plus 40 mg Vitamin B6 sowie $30 \mu \mathrm{g}$ Cyanocobalamin, gefolgt von einer erneuten Bestimmung des tHcy-Spiegels nach einem Monat. Patienten mit mittlerer (30-100 $\mu \mathrm{mol} / \mathrm{l})$ oder schwerer (>100 $\mu \mathrm{mol} / \mathrm{l})$ Hyperhomocysteinämie sowie Patienten, welche auf die Therapie nicht ansprechen, sollten an einen Spezialisten überwiesen werden. 


\section{Hämostatische Risikofaktoren für kardio- vaskuläre Krankheiten: D-Dimer}

W. Korte, W. F. Riesen

\section{Wissenschaftliche Grundlagen}

Die Entwicklung einer Atherothrombose hängt unter anderem von prokoagulatorischen Faktoren ab. Vor allem in den letzten Jahren wurde untersucht, ob Veränderungen im Gerinnungssystem Risikofaktoren für Koronarsyndrome sein könnten. Konsistente, klinisch relevante Daten existieren jedoch bislang nur für Fibrinogen und D-Dimer. Da durch die Aktivierung der Gerinnung Fibrinogen in Fibrin umgewandelt wird, stellen Marker der Fibrinbildung wie D-Dimer und des folgenden Fibrinabbaus einen «fibrinabhängigen» Risikofaktor dar im Gegensatz zu Fibrinogen. D-Dimer entsteht aus quervernetztem Fibrin nach proteolytischem Abbau durch Plasmin, so dass seine Konzentration zu einem bestimmten Zeitpunkt hauptsächlich von der Menge des gebildeten Fibrins abhängt. Eine erhöhte D-Dimer-Konzentration bei klinisch gesunden Männern einer Zufallspopulation ist mit einem erhöhten Risiko für das spätere Auftreten eines Myokardinfarkts, einer koronaren Herzkrankheit und vaskulärer Ereignisse verbunden (bei Fehlen einer Akutphasenreaktion, beispielsweise einer Infektion und/oder einem thrombotischen oder embolischen Krankheitsbild). Derzeit existieren jedoch noch keine Daten über den Nutzen eines D-Dimer-Screenings in der Allgemeinbevölkerung, im Vergleich zum Screening anderer allgemein anerkannter Risikofaktoren.

Für ein Frührezidiv eines akuten Koronarsyndroms haben sich D-Dimer-Konzentrationen im oberen Quartil als unabhängiger Risikofaktor erwiesen. Darüber hinaus existiert eine Assoziation zwischen erhöhten D-Dimer-Konzentrationen und dem Vorliegen einer koronaren Herzkrankheit. Dies trifft auch nach Berücksichtigung eines Nikotinkonsums, weiterer klassischer Risikofaktoren und des sozioökonomischen Status zu - auch bei stabiler koronarer Herzkrankheit (KHK).

\section{D-Dimer-Bestimmung}

Seit einiger Zeit stehen neben präzisen automatisierten quantitativen D-Dimer-Assays neuere quantitative POCT-(«Point of Care Test»-)Assays zur Verfügung; gegenwärtig sollte die Bestimmung jedoch mit ELISA- oder ELISA-ähnlichen Verfahren erfolgen, um eine zufriedenstellende Testqualität zu garantieren.

\section{Empfehlungen}

Zusammenfassend ergeben sich aus den verfügbaren Daten zum D-Dimer als Risikofaktor folgende Konklusionen:

- Zwischen erhöhten D-Dimer-Konzentrationen einerseits sowie Entwicklung und Ausmass einer koronaren Herzkrankheit und ihren Komplikationen (besonders akutes Koronarsyndrom) andererseits existiert eine positive Assoziation, vor allem bei Männern.

- Derzeit existiert kein allgemeingültiger (auf alle Assays und verschiedene Erkrankungssituationen anwendbarer) «Cut-off»-Level, um «erhöhte» von «normalen» D-DimerKonzentrationen zu unterscheiden; vielmehr muss für jeden Assay ein separater «Cut-off»Level definiert werden.

- Aufgrund der aktuellen Datenlage erscheint es nicht sinnvoll, die D-Dimere routinemässig zur Risikostratifizierung irgendeiner Form der koronaren Herzkrankheit zu verwenden, da die D-Dimere nicht als Kriterium bei der Entscheidung dienen, ob eine spezifische Therapie gestartet werden soll oder nicht.
Dr. med. Wolfgang Korte

Institut für klinische Chemie und

Hämatologie

Kantonsspital

CH-9007 St. Gallen

E-Mail: wolfgang.korte@ikch.ch 


\section{Entzündung und Infektion als kardiovaskuläre Risikofaktoren}

F. Mach

Korrespondenz:

Prof. Dr. med. François Mach Service de cardiologie

Département Médecine interne

Rue Micheli-du-Crest 24

CH-1211 Genève

E-Mail:

francois.mach@medecine.unige.ch

\section{Entzündung: Hs-CRP}

Atherosklerotische Plaques mit erhöhtem Komplikationsrisiko enthalten grosse Mengen an Entzündungszellen (Makrophagen und T-Lymphozyten). Hs-CRP (high-sensitivity Creactive protein), ein Serummarker für systemische Entzündungen, ist bei Patienten mit koronarer Herzkrankheit erhöht und liefert wichtige Informationen über die kurz- und langfristige Prognose von Patienten mit instabiler Angina pectoris. Ausserdem sind erhöhte hs-CRP-Werte bei gesunden Männern und Frauen mit einem erhöhten Risiko für künftige kardiale Ereignisse assoziiert. Obwohl der entzündliche Prozess eine wesentliche Rolle in der Atherogenese spielt und ein potentielles Ziel für interventionelle Massnahmen darstellt, sind die primär- und sekundärpräventiven Effekte antiinflammatorischer Behandlungen bislang nicht bekannt. Es fanden sich Hinweise auf einen günstigen antiinflammatorischen Effekt von Aspirin auf die Prävention koronarer Ereignisse. Vor kurzem wurde festgestellt, dass eine Langzeittherapie mit Statinen $\mathrm{zu}$ einer LDL-unabhängigen Senkung der hs-CRP-Werte führt. Neuere Forschungsergebnisse, die einen Zusammenhang zwischen Entzündungsmarkern und kardiovaskulärem Risiko aufzeigen, sind zweifelsohne spektakulär; aufgrund der relativen Unspezifität dieser Entzündungsmarker muss ihr potentieller Nutzen in der klinischen Routine jedoch noch nachgewiesen werden. Derzeit existieren noch keine überzeugenden Beweise, dass ein Screening von Entzündungsmarkern wie hs-CRP Vorteile für die Primär- und Sekundärprävention kardiovaskulärer Erkrankungen ergibt.

\section{Infektion}

Ein möglicher Zusammenhang zwischen Infektionskrankheiten und der Entwicklung einer Atherosklerose stellt zwar eine faszinierende Perspektive dar, ist jedoch bislang weitgehend hypothetischer Natur. In verschiedenen Studien wurden bei Patienten mit koronarer Herzkrankheit erhöhte Titer von Antikörpern gegen Infektionserreger nachgewiesen; in den meisten Fällen handelte es sich dabei um das Zytomegalievirus (CMV) oder um die bakteriellen Erreger Helicobacter pylori (H. pylori) und Chlamydia pneumoniae (C. pneumoniae). Der stärkste Verdacht richtet sich gegenwärtig gegen $C$. pneumoniae. In letzter Zeit zeigte sich in verschiedenen umfangreichen klinischen Studien, an denen mehr als 5000 Patienten mit bekannter koronarer Herzkrankheit teilgenommen hatten, dass eine Antibiotikabehandlung im Vergleich zu Placebo zu keinem eindeutig positiven Effekt führte. Das Screening von Infektionskrankheiten und eine spezifische erregergerichtete antibiotische Behandlung erscheinen derzeit weder zur Primär- noch zur Sekundärprävention kardiovaskulärer Erkrankungen gerechtfertigt. 